\title{
Increasing of Resistance and Resilience of an Urban System against Calamities in the Light of the Maximum Ordinality Principle
}

\author{
Corrado Giannantoni ${ }^{1}$, Laura Cennini ${ }^{2}$ \\ ${ }^{1}$ Rome, Italy \\ ${ }^{2}$ Colli del Tronto, Ascoli Piceno, Italy \\ Email: corrado.giannantoni@tin.it
}

How to cite this paper: Giannantoni, C. and Cennini, L. (2021) Increasing of Resistance and Resilience of an Urban System against Calamities in the Light of the Maximum Ordinality Principle. Journal of Applied Mathematics and Physics, 9, 1926-1945.

https://doi.org/10.4236/jamp.2021.98126

Received: June 15, 2021

Accepted: August 16, 2021

Published: August 19, 2021

Copyright $\odot 2021$ by author(s) and Scientific Research Publishing Inc. This work is licensed under the Creative Commons Attribution International License (CC BY 4.0).

http://creativecommons.org/licenses/by/4.0/ (c) (i) Open Access

\begin{abstract}
The present paper aims at showing how it is possible to requalify the structures of an urban system, in order to increase its resistance and its correlative resilience, against natural calamities (earthquakes, hurricanes, etc.), by adopting as reference criterion the Maximum Ordinality Principle (MOP). In this sense, the paper opens a radically new perspective in this field. In fact, the village assumed as a case study was modelled as a Self-Organizing System. This is because, although the village is usually considered as being solely made of buildings, streets, places and so on, in reality it has been conceived, planned and realized by human beings during several centuries. In addition, the people who actually leave in such an urban center, systematically deal with its maintenance, in order to possibly increase its functionality. This justifies the assumption of the village as being a Self-Organizing System and, consequently, it has been analyzed in the light of the MOP, which represents a valid reference principle for analyzing both "non-living", "living" and "conscious" self-organizing systems.
\end{abstract}

\section{Keywords}

Resistance and Resilience of Urban Systems, Maximum Ordinality Principle, Incipient Derivative

\section{Introduction: The Ostensive Example Adopted as a Case Study}

The Urban System assumed as a case study is an Italian village named Montebello di Bertona, of 940 inhabitants, in the province of Pescara (Italy), located 596 meters above sea level, in the National Park of Gran Sasso. 
This village has already been object of previous studies. Consequently, for our analysis we considered as basic reference a well-known article, published in the Magazine "Urbanistica" [1], which represents an exhaustive document, based on the traditional approach, from which we have taken the fundamental data for the analysis of its seismic requalification, now in the light of the new perspective offered by the MOP.

In this respect, it is worth preliminarily recalling the basic aspects of the MOP and its correlative formal language.

\section{The Physical Principle Adopted for the Analysis and Its Correlative Formal Language}

The Maximum Ordinality Principle (MOP), presented in 2010 at the 6th Biennial Emergy Conference, Univ. of Florida [2], is a Principle that is apt to describe the "Emerging Quality" of Self-Organizing Systems. Its verbal enunciation asserts that "Every System tends to maximize its Ordinality, including that of its surrounding habitat", and it is formulated by means of two fundamental equations, which are so strictly related to each other so as to form a Whole [3].

\section{The First Fundamental Equation}

It is formulated as follows

$$
\begin{gathered}
(\underline{\tilde{d} / \tilde{d} t})_{s}^{(\tilde{m} / \tilde{n})}\{\tilde{r}\} \stackrel{[\rightarrow}{=}\{\tilde{0}\} \\
(\tilde{m} / \tilde{n}) \rightarrow \operatorname{Max} \rightarrow\{\tilde{2} / \tilde{2}\} \uparrow\{\tilde{N} / \tilde{N}\}
\end{gathered}
$$

where: $\{\tilde{r}\}$ is the Relational Space of the System under consideration, while $(\tilde{m} / \tilde{n})$ represents its corresponding Ordinality, which reaches its maximum when it equals $\{\tilde{2} / \tilde{2}\} \uparrow\{\tilde{N} / \tilde{N}\}$ (as indicated in Equation (1.1)).

In this respect, it is worth noting that:

1) The underlined symbol $(\underline{\tilde{d} / \tilde{d} t})$ s explicitly indicates that the Generative Capacity of the System (more appropriately termed as Generativity), is "internal" to the same System, precisely because it is the one which gives origin to its Self-Organization as a Whole;

2) The symbol " $=\{\tilde{0}\}$ ” represents a more general version of the simple figure "zero as the latter systematically appears in the traditional differential equations. In fact it now represents, at the same time:

- The specific "origin and habitat" conditions associated to the considered Ordinal Differential Equation (1);

- While the symbol $\stackrel{[\rightarrow}{=}$ ” indicates that the System, during its Generative Evolution, is persistently "adherent" to its "origin and habitat" conditions.

\section{The Second Fundamental Equation}

It is formulated as follows

$$
(\tilde{d} / \tilde{d} t)^{(\tilde{2} / \tilde{2})}\left\{\{\tilde{r}\} \circledast(\tilde{d} / \tilde{d} t)^{(\tilde{2} / \tilde{2})}\{\tilde{r}\}\right\}^{[\rightarrow}=\{\tilde{0}\}
$$

and it can be considered as representing a global Feed-Back Process of Ordinal 
Nature, which is internal to the same System. Equation (2), in fact, asserts that the Relational Space of the System $\{\tilde{r}\}$, which "emerges" as a solution from the First Equation, interacts in the form of the Relational Product ${ }^{\circledR}$ (defined in Appendix) with its proper Generative Capacity $(\tilde{d} / \tilde{d} t)^{(\tilde{2} / \tilde{2})}\{\tilde{r}\}$. In such a way as to originate a comprehensive Generative Capacity which, at any time, is always adherent to the origin and habitat conditions of the Second Fundamental Equation.

\section{The Correlative Formal Language}

As previously shown, the MOP is formulated in terms of a new concept of derivative, the "Incipient Derivative", which is synthetically here recalled for the sake of clarity.

Its introduction is directly referable to the fact that Self-Organizing Systems always show an unexpected "excess" with respect to their phenomenological premises. So that they usually say: "The Whole is much more than its parts".

Such an "excess" can be termed as Quality (with a capital Q) because it cannot be understood as being a simple "property" of a given phenomenon. This is because it is never reducible to its phenomenological premises in terms of traditional mental categories: efficient causality, logical necessity, functional relationships. Consequently, it cannot be described in terms of the traditional derivative that, at the level of formal language, represents the prefect reflex of such "a priori" mental categories.

This evidently suggests a radically new gnosiological perspective and, in adherence, the adoption of "new mental categories": Emerging Causality, Generative Logic, Ordinal Relationships. These, in turn, suggest the development of a completely new formal language, in order to formulate one sole Reference Principle [4], the Maximum Ordinality Principle, so that the description of Self-Organizing Systems might result as being faithfully conform to their "Emerging Quality" [5].

This is why a new concept of derivative was introduced, that is the "Incipient Derivative" [6], which is defined as

$$
\left(\frac{\tilde{d}}{\tilde{d} t}\right)^{\tilde{q}} f(t)=\underset{\tilde{\Delta} t: 00^{+}}{\operatorname{Lim}} \circ\left(\frac{\tilde{\delta}-1}{\tilde{\Delta} t}\right)^{\tilde{q}} \circ f(t) \text { for } \tilde{q}=\tilde{m} / \tilde{n}
$$

A definition which clearly shows that the "Incipient Derivative" is not an "operator", like the derivative $(d / d t)$ in the Traditional Differential Calculus

${ }^{1}$ These "new mental categories" can no longer be termed as "pre-suppositions", because they are not defined "a priori" (as in the case of Traditional Approach). In fact, they are adopted "a posteriori", only on the basis of the "Emerging Quality" previously recognized. "Emerging Causality", in fact, refers to the capacity of a Self-Organizing System to manifest an "irreducible excess"; "Generative Logic" correspondently refers to the capacity of our mind to draw "emerging conclusions". That is, "conclusions" whose information content is much higher than the information content corresponding to their logical premises, although they are persistently "adherent" to the latter. "Ordinal Relationships", in turn, refer to particular relationships of genetic nature, like in the case of "brothers". The latter in fact are termed as such not because of their "direct reciprocal relationships", but because their direct reference to the same genetic principle: their father (or their mother or both). 
(TDC), but it could be termed as a "generator", because it describes a Process in its same act of being born. In fact:

1) The sequence of the symbols is now interpreted according to the direct priority of the three elements that constitute its definition (from left to right). This is why they acquire a completely different meaning with respect to the traditional one;

2) The three symbols, in fact, do not represent "three" distinct operations, but a unique and sole Generative Process;

3) The symbol Lĩm, whose etymological origin comes from the Latin word "Limen" (which means a "threshold"), represents the "threshold" of that "ideal window" from which we observe and describe the considered phenomenon;

4) The symbol $\tilde{\Delta} t: 0 \rightarrow 0^{+}$now indicates not only the initial time of our registration, but also the proper "origin" (in its etymological sense) of something new which we observe (and describe) in its proper act of being born, as a generative process [7];

5) It is then evident that the "operator" $\tilde{\delta}$ now registers the variation of the observed property $f(t)$, not only in terms of quantity, but also, and especially, in terms of Quality (as the symbol "tilde" would expressly remind). Thus the ratio which appears in Equation (3) indicates not only a quantitative variation in time, but both the variation in Quality and quantity;

6) Consequently, when we take the incipient (or "prior") derivative of Ordinality $\tilde{q}$ of any $f(t)$, the exit of such a process will keep "memory" of its genetic origin because, besides its quantity, it will result as being structured according the indication of such an exponent. The latter in fact is properly termed as Ordinality, because it precisely expresses how each part of the output is genetically Ordered to the Whole and, at the same time, how each part is related to all the others in terms of Ordinal Relationships (as shown by Equations (A1) and (A5) in Appendix);

7) In this way the "incipient" derivative represents the Generativity of the considered Process, that is the output "excess" (per unit time) characterized by both its Ordinality and its related cardinality, while the sequence of the symbols in its definition (Equation (3)) can be interpreted as representing a unique inter-action process between the same;

8) The above-mentioned reasons clearly show why the "incipient" derivative is able to unify (and, at the same time, to specify) the description of the various Self-Organizing Processes, when explicitly understood in terms of Quality;

9) This also means that the Incipient Derivative has an exit that is generally different from the result of the corresponding derivative in TDC, even when its Ordinality is reduced to a mere cardinality. For example, the derivative of order $n$ of the function $e^{\alpha(t)}$ (evaluated according to Faà di Bruno's formula) and the corresponding incipient derivative, give

$$
\left(\frac{d}{d t}\right)^{n} e^{\alpha(t)}=e^{\alpha(t)} \sum \frac{n !}{k_{1} ! k_{2} ! \cdots k_{n} !} \cdot\left(\frac{\dot{\alpha}}{1 !}\right)^{k_{1}}\left(\frac{\ddot{\alpha}}{2 !}\right)^{k_{2}} \cdots\left(\frac{\alpha^{(n)}}{n !}\right)^{k_{n}}
$$


and

$$
\left(\frac{\tilde{d}}{\tilde{d} t}\right)^{n} e^{\alpha(t)} \stackrel{*}{=} e^{\alpha(t)} \cdot[\stackrel{\circ}{\alpha}(t)]^{n}
$$

respectively, where $\alpha(t)$ represents the first order incipient derivative. And even if in some cases they coincide (for instance when $\alpha(t)$ is linear) such a coincidence has to be seen in the light of the symbol = in Equation (5), which reminds us that any incipient derivative is always the exit of a generative logical process and not of a necessary logical process.

\section{Description of the Urban System Analyzed}

The description of the village is based on the Analysis of the so called "Minimun Urban Structure" which, as analyzed by V. Fabietti and Others [1], is composed by 39 elements (buildings, roads, utilities). So that, according to the available data [ib.], it can be characterized by means of three Indicators: Vulnerability, Index of Urban Complex, Strategic Valence.

The "Vulnerability" is the probability that a threat event can induce a more or less marked loss of integrity.

The "Index of Urban Complex" (or, more simply, "Index of Complex") represents the level of physical and functional interconnections with neighboring elements.

The "Strategic Valence", according to a conventional scale from 1 to 12, indicates the relevance of the role assigned to each specific element, for example: administrative center, hospital, commercial center and so on.

It is worth adding that, for practical reasons, in our analysis we have also adopted the inverse concept of "Vulnerability", defined as "Persistence to Integrity" (or, more synthetically, "Persistence").

The physical properties of the 39 elements of the village, expressed in terms of such Indicators, are shown in Table 1.

\subsection{Reconfiguration of the Village in Ordinal Terms}

The Reconfiguration of the village was obtained by means a Simulator, termed as EQS (Emerging Quality Simulator), which is based on the explicit solution to the MOP (see Appendix), and thus it is not conceived as a traditional computer code that operates in functional terms, but on the basis of Ordinal Relationships between the various elements of the village.

The specific Ordinal Reconfiguration of the village, modelled (as previously said) as a Self-Organizing System, was researched for in such a way as to keep invariant the values of the Indicators corresponding to element 39, which in particular, apart from its other properties, is characterized by the maximum value of Persistence. A choice that could possibly correspond to the minimum costs and times of the Requalification Process when operatively realized.

The Ordinal properties of the village, after such an Ordinal Reconfiguration, are shown in Table 2, from which is possible to recognize that the values 
Table 1. The real urban system and its 39 elements, rearranged for increasing persistence.

\begin{tabular}{|c|c|c|c|c|c|}
\hline $\begin{array}{l}\text { Progressive } \\
\text { Number }\end{array}$ & $\begin{array}{l}\text { Number of the } \\
\text { Real Element }\end{array}$ & Persistence & Vulnerability & $\begin{array}{l}\text { Index of } \\
\text { Complex }\end{array}$ & $\begin{array}{l}\text { Strategic } \\
\text { Valence }\end{array}$ \\
\hline 1 & 4 & 2.000 & 0.5000 & 1.000 & 1.000 \\
\hline 3 & 18 & 2.170 & 0.4608 & 3.800 & 2.250 \\
\hline 4 & 6 & 2.220 & 0.4505 & 1.000 & 1.100 \\
\hline 5 & 15 & 2.220 & 0.4505 & 3.300 & 2.040 \\
\hline 6 & 17 & 2.220 & 0.4505 & 3.300 & 2.050 \\
\hline 7 & 8 & 2.220 & 0.4505 & 4.400 & 2.750 \\
\hline 8 & 19 & 2.220 & 0.4505 & 4.800 & 3.940 \\
\hline 9 & 3 & 2.220 & 0.4505 & 3.100 & 2.100 \\
\hline 10 & 14 & 2.270 & 0.4405 & 3.300 & 2.060 \\
\hline 11 & 16 & 2.270 & 0.4405 & 3.200 & 2.060 \\
\hline 12 & 10 & 2.270 & 0.4405 & 4.200 & 2.700 \\
\hline 13 & 9 & 2.270 & 0.4405 & 4.300 & 2.730 \\
\hline 14 & 20 & 2.270 & 0.4405 & 5.300 & 4.560 \\
\hline 15 & 11 & 2.325 & 0.4301 & 10.900 & 7.440 \\
\hline 16 & 21 & 2.325 & 0.4301 & 5.300 & 4.560 \\
\hline 17 & 12 & 2.380 & 0.4202 & 10.800 & 7.420 \\
\hline 18 & 13 & 2.439 & 0.4100 & 10.700 & 7.400 \\
\hline 19 & 1 & 2.500 & 0.4000 & 1.000 & 1.200 \\
\hline 20 & 7 & 2.500 & 0.4000 & 5.900 & 4.625 \\
\hline 21 & 39 & 2.500 & 0.4000 & 11.800 & 9.250 \\
\hline 22 & 2 & 2.860 & 0.3497 & 1.000 & 1.200 \\
\hline 23 & 5 & 2.940 & 0.3401 & 1.000 & 1.500 \\
\hline 24 & 38 & 3.330 & 0.3003 & 9.900 & 6.180 \\
\hline 25 & 34 & 3.700 & 0.2703 & 7.000 & 5.310 \\
\hline 26 & 35 & 4.000 & 0.2500 & 7.000 & 6.000 \\
\hline 27 & 36 & 4.000 & 0.2500 & 7.000 & 6.000 \\
\hline 28 & 28 & 4.348 & 0.2300 & 9.100 & 7.250 \\
\hline 29 & 37 & 4.545 & 0.2200 & 9.200 & 6.680 \\
\hline 30 & 30 & 4.545 & 0.2200 & 9.500 & 7.850 \\
\hline 31 & 33 & 4.545 & 0.2200 & 12.900 & 10.560 \\
\hline 32 & 29 & 4.760 & 0.2101 & 9.600 & 7.870 \\
\hline 33 & 25 & 5.000 & 0.2000 & 8.000 & 10.000 \\
\hline 34 & 26 & 5.000 & 0.2000 & 8.500 & 10.000 \\
\hline 35 & 31 & 5.000 & 0.2000 & 12.200 & 9.310 \\
\hline 36 & 32 & 5.000 & 0.2000 & 12.200 & 9.290 \\
\hline 37 & 27 & 5.000 & 0.2000 & 13.900 & 13.900 \\
\hline 38 & 24 & 6.660 & 0.1502 & 7.000 & 12.000 \\
\hline 39 & 23 & 6.660 & 0.1502 & 7.500 & 12.000 \\
\hline
\end{tabular}


Table 2. Ordinal requalification of the urban system articulated in its 39 elements.

\begin{tabular}{|c|c|c|c|c|c|}
\hline $\begin{array}{l}\text { Progressive } \\
\text { Number }\end{array}$ & $\begin{array}{l}\text { Number of the } \\
\text { Real Element }\end{array}$ & Persistence & Vulnerability & $\begin{array}{l}\text { Index of } \\
\text { Complex }\end{array}$ & $\begin{array}{l}\text { Strategic } \\
\text { Valence }\end{array}$ \\
\hline 1 & 4 & 2.0000 & 0.5000 & 1.0000 & 1.0000 \\
\hline 2 & 22 & 2.7847 & 0.3591 & 2.0922 & 1.3116 \\
\hline 3 & 18 & 2.8234 & 0.3542 & 3.0051 & 1.6003 \\
\hline 4 & 6 & 2.8639 & 0.3492 & 3.8297 & 1.8890 \\
\hline 5 & 15 & 2.9065 & 0.3441 & 4.5725 & 2.1777 \\
\hline 6 & 17 & 2.9512 & 0.3388 & 5.2393 & 2.4664 \\
\hline 7 & 8 & 2.9981 & 0.3335 & 5.8355 & 2.7551 \\
\hline 8 & 19 & 3.0473 & 0.3282 & 6.3664 & 3.0438 \\
\hline 9 & 3 & 3.0989 & 0.3227 & 6.8367 & 3.3325 \\
\hline 10 & 14 & 3.1531 & 0.3171 & 7.2510 & 3.6212 \\
\hline 11 & 16 & 3.2099 & 0.3115 & 7.6135 & 3.9099 \\
\hline 12 & 10 & 3.2696 & 0.3058 & 7.9281 & 4.1986 \\
\hline 13 & 9 & 3.3322 & 0.3001 & 8.1985 & 4.4873 \\
\hline 14 & 20 & 3.3978 & 0.2943 & 8.4283 & 4.7760 \\
\hline 15 & 11 & 3.4667 & 0.2885 & 8.6206 & 5.0647 \\
\hline 16 & 21 & 3.5390 & 0.2826 & 8.7784 & 5.3534 \\
\hline 17 & 12 & 3.6149 & 0.2766 & 8.9046 & 5.6421 \\
\hline 18 & 13 & 3.6945 & 0.2707 & 9.0018 & 5.9308 \\
\hline 19 & 1 & 3.7780 & 0.2647 & 9.0724 & 6.2194 \\
\hline 20 & 7 & 3.8657 & 0.2587 & 9.1187 & 6.5081 \\
\hline 21 & 39 & 3.9577 & 0.2527 & 9.1428 & 6.7968 \\
\hline 22 & 2 & 4.0542 & 0.2467 & 9.1468 & 7.0855 \\
\hline 23 & 5 & 4.1554 & 0.2407 & 9.1324 & 7.3742 \\
\hline 24 & 38 & 4.2617 & 0.2346 & 9.1014 & 7.6629 \\
\hline 25 & 34 & 4.3732 & 0.2287 & 9.0553 & 7.9516 \\
\hline 26 & 35 & 4.4901 & 0.2227 & 8.9957 & 8.2403 \\
\hline 27 & 36 & 4.6129 & 0.2168 & 8.9240 & 8.5290 \\
\hline 28 & 28 & 4.7417 & 0.2109 & 8.8413 & 8.8177 \\
\hline 29 & 37 & 4.8768 & 0.2051 & 8.7489 & 9.1064 \\
\hline 30 & 30 & 5.0186 & 0.1993 & 8.6479 & 9.3951 \\
\hline 31 & 33 & 5.1674 & 0.1935 & 8.5392 & 9.6838 \\
\hline 32 & 29 & 5.3236 & 0.1878 & 8.4239 & 9.9725 \\
\hline 33 & 25 & 5.4874 & 0.1822 & 8.3028 & 10.2612 \\
\hline 34 & 26 & 5.6593 & 0.1767 & 8.1767 & 10.5499 \\
\hline 35 & 31 & 5.8397 & 0.1712 & 8.0463 & 10.8386 \\
\hline 36 & 32 & 6.0289 & 0.1659 & 7.9124 & 11.1273 \\
\hline 37 & 27 & 6.2275 & 0.1606 & 7.7755 & 11.4160 \\
\hline 38 & 24 & 6.4359 & 0.1554 & 7.6361 & 11.7047 \\
\hline 39 & 23 & 6.6546 & 0.1503 & 7.4949 & 11.9934 \\
\hline
\end{tabular}


pertaining to element $39(6.6546,0.1503,7.4949$ respectively) are almost equal to the corresponding values of the same element in previous Table 1 (6.6600, $0.1502,7.5000)$.

Such an aspect is explicitly pointed out for two fundamental reasons: 1) first of all in order to show that the Reconfiguration Ordinal Process leaves "invariant" the best properties of the System; 2) secondly, because the Ordinal Reconfiguration represented in Table 2 was obtained through three intermediate passages:

- A preliminary representation of the real village in terms of "couples" of elements.

- The research for a similar reconfiguration, by means of the Simulator EQS, in terms of Ordinal "Duets".

- A final disarticulation of the Ordinal "Duets" in terms of "single" elements.

This is because, properly speaking, in an Ordinal context there are not "single" elements, because they are always related to each other in Ordinal terms, and the minimum level of Ordinality is exactly that represented by Ordinal " $\mathrm{Du}$ ets".

\subsection{Requalification of the Village in Operative Terms}

The comparison between Table 1 and Table 2 shows that several Indicators of the real village have to be re-qualified in order to transform the village into an Ordinal Self-Organizing System.

The Indicators that should specifically be re-qualified are shown in Table 3, in which:

- The values of the Indicators without any specific indication do not necessitate to be re-qualified.

- The values of the Indicators that are market by an asterisk are all to be to be re-qualified to assume the same values as those indicated in Table 2.

In particular, the values of Strategic Valence that have not any specific indication do not necessitate to be updated.

- The values which are marked by $\left(^{*}\right)$, even if apparently seem to require a re-qualification (as usually indicated by an asterisk), in reality do not necessitate to be updated (and this is the meaning of the "brackets" ( )). This is because in an Ordinal Context, among "Persistence", "Index of Complex" and "Strategic Valence" pertaining to each specific element, there exists an Ordinal Harmony Relationship which, when expressed in terms of its reflexed cardinalities, asserts that

"Persistence" $\times$ "Index of Complex" $=$ “ $\lambda$ " $\times$ "Strategic Valence"

where " $\lambda$ " is a constant which is characteristic of the Ordinal System analyzed. Consequently, the values of the "Strategic Valence" can be considered as being contextually re-qualified as a direct consequence of the re-qualification of the other two correlative Indicators. 
Table 3. "Ordinal requalification" of the urban system in operative terms.

\begin{tabular}{|c|c|c|c|c|c|}
\hline $\begin{array}{l}\text { Progressive } \\
\text { Number }\end{array}$ & $\begin{array}{l}\text { Number of the } \\
\text { Real Element }\end{array}$ & Persistence & Vulnerability & $\begin{array}{l}\text { Index of } \\
\text { Complex }\end{array}$ & $\begin{array}{l}\text { Strategic } \\
\text { Valence }\end{array}$ \\
\hline 1 & 4 & 2.0000 & 0.5000 & 1.0000 & 1.0000 \\
\hline 2 & 22 & $* 2.1700$ & 0.4608 & 2.2000 & $1.1000\left(^{*}\right)$ \\
\hline 3 & 18 & * 2.1700 & 0.4608 & 3.8000 & 2.2500 \\
\hline 4 & 6 & $* 2.2200$ & 0.4505 & $\star 1.0000$ & $1.1000\left(^{*}\right)$ \\
\hline 5 & 15 & $\star 2.2200$ & 0.4505 & * 3.3000 & $2.0400\left(^{*}\right)$ \\
\hline 6 & 17 & $\star 2.2200$ & 0.4505 & *3.3000 & $2.0500\left(^{*}\right)$ \\
\hline 7 & 8 & $\star 2.2200$ & 0.4505 & $\star 4.4000$ & $2.7500\left(^{\star}\right)$ \\
\hline 8 & 19 & $* 2.2200$ & 0.4505 & $* 4.8000$ & 3.9400 \\
\hline 9 & 3 & $\star 2.2200$ & 0.4505 & $* 3.1000$ & $2.1000\left(^{*}\right)$ \\
\hline 10 & 14 & $\star 2.2700$ & 0.4405 & * 3.3000 & $2.0600\left(^{*}\right)$ \\
\hline 11 & 16 & $\star 2.2700$ & 0.4405 & $* 3.2000$ & $\left.2.0600{ }^{*}\right)$ \\
\hline 12 & 10 & $\star 2.2700$ & 0.4405 & $* 4.2000$ & $2.7000\left(^{*}\right)$ \\
\hline 13 & 9 & $* 2.2700$ & 0.4405 & $* 4.3000$ & $2.7300\left(^{*}\right)$ \\
\hline 14 & 20 & *2.2700 & 0.4405 & * 5.3000 & $4.5600\left(^{*}\right)$ \\
\hline 15 & 11 & $* 2.3250$ & 0.4301 & 10.9000 & 7.4400 \\
\hline 16 & 21 & $* 2.3250$ & 0.4301 & * 5.3000 & $4.5600\left(^{\star}\right)$ \\
\hline 17 & 12 & $* 2.3800$ & 0.4202 & 10.8000 & 7.4200 \\
\hline 18 & 13 & * 2.4390 & 0.4100 & 10.7000 & 7.4000 \\
\hline 19 & 1 & $\star 2.5000$ & 0.4000 & $* 1.0000$ & $\left.1.2000{ }^{*}\right)$ \\
\hline 20 & 7 & $* 2.5000$ & 0.4000 & * 5.9000 & $4.6250\left(^{*}\right)$ \\
\hline 21 & 39 & * 2.5000 & 0.4000 & 11.8000 & 9.2500 \\
\hline 22 & 2 & $* 2.8600$ & 0.3497 & $* 1.0000$ & $\left.1.2000{ }^{*}\right)$ \\
\hline 23 & 5 & *2.9400 & 0.3401 & * 1.0000 & $1.5000\left(^{\star}\right)$ \\
\hline 24 & 38 & * 3.3300 & 0.3003 & 9.9000 & $6.1800\left(^{*}\right)$ \\
\hline 25 & 34 & * 3.7000 & 0.2703 & * 7.0000 & $5.3100\left(^{\star}\right)$ \\
\hline 26 & 35 & $* 4.0000$ & 0.2500 & * 7.0000 & $6.0000\left(^{*}\right)$ \\
\hline 27 & 36 & $\star 4.0000$ & 0.2500 & * 7.0000 & $6.0000\left(^{*}\right)$ \\
\hline 28 & 28 & * 4.3480 & 0.2300 & 9.1000 & $7.2500\left(^{*}\right)$ \\
\hline 29 & 37 & $\star 4.5450$ & 0.2200 & 9.2000 & $6.6800\left(^{*}\right)$ \\
\hline 30 & 30 & * 4.5450 & 0.2200 & 9.5000 & $7.8500\left(^{*}\right)$ \\
\hline 31 & 33 & $* 4.5450$ & 0.2200 & 12.9000 & 10.5600 \\
\hline 32 & 29 & * 4.7600 & 0.2101 & 9.6000 & $\left.7.8700{ }^{*}\right)$ \\
\hline 33 & 25 & $* 5.0000$ & 0.2000 & * 8.0000 & $10.0000\left(^{*}\right)$ \\
\hline 34 & 26 & * 5.0000 & 0.2000 & 8.5000 & $10.0000\left(^{*}\right)$ \\
\hline 35 & 31 & * 5.0000 & 0.2000 & 12.2000 & $9.3100\left(^{*}\right)$ \\
\hline 36 & 32 & $* 5.0000$ & 0.2000 & 12.2000 & $9.2900\left(^{*}\right)$ \\
\hline 37 & 27 & * 5.0000 & 0.2000 & 13.9000 & 13.9000 \\
\hline 38 & 24 & 6.6600 & 0.1502 & $* 7.0000$ & 12.0000 \\
\hline 39 & 23 & 6.6600 & 0.1502 & 7.5000 & 12.0000 \\
\hline
\end{tabular}




\subsection{The Relevance of the Ordinal Requalification Process}

At this stage one could ask: why is it worth requalifying an Urban System in Ordinal Terms?

The answer is very simple: such an Ordinal Process, in fact, will surely increase the Resistance of the Urban System against Calamities, as we will show in the next paragraphs and, at the same time, it will improve its correlative Resilience.

However, it is worth anticipating that this is true even in the case of a partial Requalification of the System. A calamity, in fact, might occur even during the Requalification Process of the System (because it could probably last some years). In such a case, the System will not correspondently be at its Maximum Ordinality. However, Equations (A5) (in Appendix) clearly show that even a partial Requalification of a given number of elements is able to give a significant contribution to the Ordinality of the System. In fact, such a contribution is always effectively higher than that estimated on the sole basis of their partial requalification level. This is because the partially re-qualified elements are always in Ordinal Relationships between them (although at a reduced level of Ordinality) and, in particular, they are in Ordinal Relationships with all the other elements already completely re-qualified, even if, the "emerging" Ordinality from all such Relationships is still not perfectly corresponding to the Maximum Ordinality. This also means that who will operatively realize the Requalification, even if he does not know the MOP and thus interprets the values of the Indicators as being "separated" cardinal entities, in reality, by requalifying any single cardinal value, he will operatively realize an "accelerated" Ordinal Requalification of the System as a Whole.

\section{Description of the Considered Calamity and Its Potential Effects on the Urban System}

The evaluation of the Resistance of the System was obtained by simulating by means of EQS, and in conformity to Equation (2), the Ordinal Inter-Action between the System in its re-qualified Ordinal configuration and the Ordinal configuration of the same after the estimated effects due to a foreseeable calamity.

In adherence to the MOP, the analysis is then performed by modelling both the two configurations of the System in terms of "couples" of elements (or better "Duets"), for the same reasons previously recalled. Consequently, the Reference Ordinal Structure of the System is shown in Table 4, where the values of Duets are referred, by difference, to element 1 .

As far as the calamity is concerned, we assumed, as a work hypothesis, an earthquake characterized by:

- An "incidence" of the order of $5 \%$ on the value of high Persistence and of $10 \%$ on that at low Persistence.

- And similar incidence values with respect to the other two correlative Indicators. 
Table 4. Ordinal requalification of the urban system considered as being made up of 38 couples of elements.

\begin{tabular}{|c|c|c|c|c|c|c|c|c|c|}
\hline & $\mathrm{Bl}$ & $\mathrm{Cl}$ & $\mathrm{El}$ & $\rho_{1 j\left(t_{0}\right)}$ & $\varphi_{1 j\left(t_{0}\right)}$ & $\theta_{1 j\left(t_{0}\right)}$ & $\rho_{1 j\left(t_{0}+\Delta t\right)}^{\circ}$ & $\varphi_{1 j\left(t_{0}+\Delta t\right)}^{\circ}$ & $\theta_{1 j\left(t_{0}+\Delta t\right)}^{\circ}$ \\
\hline 1 & 1 & 0 & 0.3570 & 0.7847 & 1.0922 & 0.3116 & 0 & 0 & 0 \\
\hline 2 & 1 & 0 & 0.6877 & 0.8234 & 2.0051 & 0.6003 & 0 & 0 & 0 \\
\hline 3 & 1 & 0 & 1.0183 & 0.8639 & 2.8297 & 0.8890 & 0 & 0 & 0 \\
\hline 4 & 1 & 0 & 1.3490 & 0.9065 & 3.5725 & 1.1777 & 0 & 0 & 0 \\
\hline 5 & 1 & 0 & 1.6797 & 0.9512 & 4.2393 & 1.4664 & 0 & 0 & 0 \\
\hline 6 & 1 & 0 & 2.0104 & 0.9981 & 4.8355 & 1.7551 & 0 & 0 & 0 \\
\hline 7 & 1 & 0 & 2.3411 & 1.0473 & 5.3664 & 2.0438 & 0 & 0 & 0 \\
\hline 8 & 1 & 0 & 2.6718 & 1.0989 & 5.8367 & 2.3325 & 0 & 0 & 0 \\
\hline 9 & 1 & 0 & 3.0025 & 1.1531 & 6.2510 & 2.6212 & 0 & 0 & 0 \\
\hline 10 & 1 & 0 & 3.3332 & 1.2099 & 6.6135 & 2.9099 & 0 & 0 & 0 \\
\hline 11 & 1 & 0 & 3.6639 & 1.2696 & 6.9281 & 3.1986 & 0 & 0 & 0 \\
\hline 12 & 1 & 0 & 3.9946 & 1.3322 & 7.1985 & 3.4873 & 0 & 0 & 0 \\
\hline 13 & 1 & 0 & 4.3253 & 1.3978 & 7.4283 & 3.7760 & 0 & 0 & 0 \\
\hline 14 & 1 & 0 & 4.6560 & 1.4667 & 7.6206 & 4.0647 & 0 & 0 & 0 \\
\hline 15 & 1 & 0 & 4.9867 & 1.5390 & 7.7784 & 4.3534 & 0 & 0 & 0 \\
\hline 16 & 1 & 0 & 5.3174 & 1.6149 & 7.9046 & 4.6421 & 0 & 0 & 0 \\
\hline 17 & 1 & 0 & 5.6481 & 1.6945 & 8.0018 & 4.9308 & 0 & 0 & 0 \\
\hline 18 & 1 & 0 & 5.9788 & 1.7780 & 8.0724 & 5.2194 & 0 & 0 & 0 \\
\hline 19 & 1 & 0 & 6.3095 & 1.8657 & 8.1187 & 5.5081 & 0 & 0 & 0 \\
\hline 20 & 1 & 0 & 6.6401 & 1.9577 & 8.1428 & 5.7968 & 0 & 0 & 0 \\
\hline 21 & 1 & 0 & 6.9708 & 2.0542 & 8.1468 & 6.0855 & 0 & 0 & 0 \\
\hline 22 & 1 & 0 & 7.3015 & 2.1554 & 8.1324 & 6.3742 & 0 & 0 & 0 \\
\hline 23 & 1 & 0 & 7.6322 & 2.2617 & 8.1014 & 6.6629 & 0 & 0 & 0 \\
\hline 24 & 1 & 0 & 7.9629 & 2.3732 & 8.0553 & 6.9516 & 0 & 0 & 0 \\
\hline 25 & 1 & 0 & 8.2936 & 2.4901 & 7.9957 & 7.2403 & 0 & 0 & 0 \\
\hline 26 & 1 & 0 & 8.6243 & 2.6129 & 7.9240 & 7.5290 & 0 & 0 & 0 \\
\hline 27 & 1 & 0 & 8.9550 & 2.7417 & 7.8413 & 7.8177 & 0 & 0 & 0 \\
\hline 28 & 1 & 0 & 9.2857 & 2.8768 & 7.7489 & 8.1064 & 0 & 0 & 0 \\
\hline 29 & 1 & 0 & 9.6164 & 3.0186 & 7.6479 & 8.3951 & 0 & 0 & 0 \\
\hline 30 & 1 & 0 & 9.9471 & 3.1674 & 7.5392 & 8.6838 & 0 & 0 & 0 \\
\hline 31 & 1 & 0 & 10.2778 & 3.3236 & 7.4239 & 8.9725 & 0 & 0 & 0 \\
\hline 32 & 1 & 0 & 10.6085 & 3.4874 & 7.3028 & 9.2612 & 0 & 0 & 0 \\
\hline 33 & 1 & 0 & 10.9392 & 3.6593 & 7.1767 & 9.5499 & 0 & 0 & 0 \\
\hline 34 & 1 & 0 & 11.2699 & 3.8397 & 7.0463 & 9.8386 & 0 & 0 & 0 \\
\hline 35 & 1 & 0 & 11.6006 & 4.0289 & 6.9124 & 10.1273 & 0 & 0 & 0 \\
\hline
\end{tabular}




\section{Continued}

\begin{tabular}{llllllllll}
\hline 36 & 1 & 0 & 11.9312 & 4.2275 & 6.7755 & 10.4160 & 0 & 0 & 0 \\
37 & 1 & 0 & 12.2619 & 4.4359 & 6.6361 & 10.7047 & 0 & 0 & 0 \\
38 & 1 & 0 & 12.5926 & 4.6546 & 6.4949 & 10.9934 & 0 & 0 & 0
\end{tabular}

Captions: $\mathrm{Bl}, \mathrm{Cl}, \mathrm{Dl}$ are internal control parameters of the simulator EQS; $\rho_{1 j\left(t_{0}\right)}, \varphi_{1 j\left(t_{0}\right)}, \theta_{1 j\left(t_{0}\right)}$ represent the actual Relational Space (Persistence, index of complex, strategic valence) of each Duet at the initial time; $\rho_{1 j\left(t_{0}+\Delta t\right)}^{\circ}, \varphi_{1 j\left(t_{0}+\Delta t\right)}^{\circ}, \theta_{1 j\left(t_{0}+\Delta t\right)}^{\circ}$ are their corresponding Incipient Derivatives.

The corresponding effects of such a calamity on the System, simulated by means of EQS, are represented in Table 5.

As it is easy to recognize, the "incidence" is equal to $4.82 \%$ for the element at high Persistence (with respect to the hypothesis of 5\%), while it is equal to $9.92 \%$ for the element at low Persistence (with respect to the hypothesis of $10 \%$ ).

The effect on the "Strategic Valence", always with reference to the element at high Persistence, is equal to (5.49\%), and it is comparable with that on Persistence, while the effect on the "Index of Complex" is almost twice as much: $11.54 \%$.

This reveals a particular "sensitivity" of the "Duet" Ordinal Relationships especially for those elements that are characterized by high values of the Index of Complex.

As far as the values of the Indicators at low Persistence are concerned, apart from the value of $9.92 \%$ pertaining to the Indicator of Persistence previously mentioned, there are lower effects on the other two correlative Indicators.

This means that the elements characterized by low values of Persistence are less sensitive to the variations with respect to their correlative values of both Index of Complex and Strategic Valence.

\section{Formal Translation of the Inter-Action Previously Described by Means of the Simulator EQS}

The evolution of the process, described by the two fundamental Equations (1) and (2) of the MOP, was represented by means of EQS through three distinct successive processes:

1) The simulation of the Ordinal Reconfiguration of the System in its original integrity, as it appears in Table 4, that is considered in the absence of any external effects, and specifically structured in terms of "Duet" elements, as previously said;

2) The simulation of the Inter-Action of the System with the hypothesized earthquake and its consequential reconfiguration, still in terms of "Duet" buildings, as represented in Table 5;

3) The simulation of the Ordinal Interaction between the configuration of the System under condition a) and the System under condition b). This is because such an Inter-Action is precisely that which reflects the proper meaning of Equation (2). 
Table 5. Ordinal Requalification of the Urban System as a consequence of the hypothesized earthquake.

\begin{tabular}{|c|c|c|c|c|c|c|c|c|c|}
\hline & $\mathrm{Bl}$ & $\mathrm{Cl}$ & $\mathrm{El}$ & $\rho_{1 j\left(t_{0}\right)}$ & $\varphi_{1 j\left(t_{0}\right)}$ & $\theta_{1 j\left(t_{0}\right)}$ & $\rho_{1 j\left(t_{0}+\Delta t\right)}^{\circ}$ & $\varphi_{1 j\left(t_{0}+\Delta t\right)}^{\circ}$ & $\theta_{1 j\left(t_{0}+\Delta t\right)}^{\circ}$ \\
\hline 1 & 1 & 0 & 0.3570 & 0.7068 & 0.1020 & 0.2945 & 0 & 0 & 0 \\
\hline 2 & 1 & 0 & 0.6877 & 0.7428 & 0.1871 & 0.5673 & 0 & 0 & 0 \\
\hline 3 & 1 & 0 & 1.0183 & 0.7805 & 0.2637 & 0.8401 & 0 & 0 & 0 \\
\hline 4 & 1 & 0 & 1.3490 & 0.8202 & 0.3324 & 1.1130 & 0 & 0 & 0 \\
\hline 5 & 1 & 0 & 1.6797 & 0.8619 & 0.3938 & 1.3858 & 0 & 0 & 0 \\
\hline 6 & 1 & 0 & 2.0104 & 0.9058 & 0.4486 & 1.6586 & 0 & 0 & 0 \\
\hline 7 & 1 & 0 & 2.3411 & 0.9518 & 0.4971 & 1.9314 & 0 & 0 & 0 \\
\hline 8 & 1 & 0 & 2.6718 & 1.0003 & 0.5398 & 2.2042 & 0 & 0 & 0 \\
\hline 9 & 1 & 0 & 3.0025 & 1.0511 & 0.5773 & 2.4771 & 0 & 0 & 0 \\
\hline 10 & 1 & 0 & 3.3332 & 1.1046 & 0.6099 & 2.7499 & 0 & 0 & 0 \\
\hline 11 & 1 & 0 & 3.6639 & 1.1608 & 0.6379 & 3.0227 & 0 & 0 & 0 \\
\hline 12 & 1 & 0 & 3.9946 & 1.2198 & 0.6619 & 3.2955 & 0 & 0 & 0 \\
\hline 13 & 1 & 0 & 4.3253 & 1.2818 & 0.6820 & 3.5684 & 0 & 0 & 0 \\
\hline 14 & 1 & 0 & 4.6560 & 1.3470 & 0.6986 & 3.8412 & 0 & 0 & 0 \\
\hline 15 & 1 & 0 & 4.9867 & 1.4155 & 0.7120 & 4.1140 & 0 & 0 & 0 \\
\hline 16 & 1 & 0 & 5.3174 & 1.4875 & 0.7225 & 4.3868 & 0 & 0 & 0 \\
\hline 17 & 1 & 0 & 5.6481 & 1.5632 & 0.7303 & 4.6596 & 0 & 0 & 0 \\
\hline 18 & 1 & 0 & 5.9788 & 1.6427 & 0.7356 & 4.9325 & 0 & 0 & 0 \\
\hline 19 & 1 & 0 & 6.3095 & 1.7262 & 0.7387 & 5.2053 & 0 & 0 & 0 \\
\hline 20 & 1 & 0 & 6.6401 & 1.8140 & 0.7398 & 5.4781 & 0 & 0 & 0 \\
\hline 21 & 1 & 0 & 6.9708 & 1.9062 & 0.7391 & 5.7509 & 0 & 0 & 0 \\
\hline 22 & 1 & 0 & 7.3015 & 2.0032 & 0.7367 & 6.0238 & 0 & 0 & 0 \\
\hline 23 & 1 & 0 & 7.6322 & 2.1051 & 0.7328 & 6.2966 & 0 & 0 & 0 \\
\hline 24 & 1 & 0 & 7.9629 & 2.2121 & 0.7275 & 6.5694 & 0 & 0 & 0 \\
\hline 25 & 1 & 0 & 8.2936 & 2.3246 & 0.7211 & 6.8422 & 0 & 0 & 0 \\
\hline 26 & 1 & 0 & 8.6243 & 2.4428 & 0.7135 & 7.1150 & 0 & 0 & 0 \\
\hline 27 & 1 & 0 & 8.9550 & 2.5671 & 0.7050 & 7.3879 & 0 & 0 & 0 \\
\hline 28 & 1 & 0 & 9.2857 & 2.6976 & 0.6957 & 7.6607 & 0 & 0 & 0 \\
\hline 29 & 1 & 0 & 9.6164 & 2.8348 & 0.6856 & 7.9335 & 0 & 0 & 0 \\
\hline 30 & 1 & 0 & 9.9471 & 2.9790 & 0.6749 & 8.2063 & 0 & 0 & 0 \\
\hline 31 & 1 & 0 & 10.2778 & 3.1305 & 0.6635 & 8.4792 & 0 & 0 & 0 \\
\hline 32 & 1 & 0 & 10.6085 & 3.2897 & 0.6518 & 8.7520 & 0 & 0 & 0 \\
\hline 33 & 1 & 0 & 10.9392 & 3.4570 & 0.6395 & 9.0248 & 0 & 0 & 0 \\
\hline 34 & 1 & 0 & 11.2699 & 3.6328 & 0.6270 & 9.2976 & 0 & 0 & 0 \\
\hline 35 & 1 & 0 & 11.6006 & 3.8175 & 0.6142 & 9.5704 & 0 & 0 & 0 \\
\hline
\end{tabular}




\section{Continued}

\begin{tabular}{llllllllll}
\hline 36 & 1 & 0 & 11.9312 & 4.0117 & 0.6011 & 9.8433 & 0 & 0 & 0 \\
37 & 1 & 0 & 12.2619 & 4.2157 & 0.5879 & 10.1161 & 0 & 0 & 0 \\
38 & 1 & 0 & 12.5926 & 4.4301 & 0.5745 & 10.3889 & 0 & 0 & 0 \\
\hline
\end{tabular}

Captions: $\mathrm{Bl}, \mathrm{Cl}, \mathrm{Dl}$ are internal control parameters of the simulator EQS; $\rho_{1 j\left(t_{0}\right)}, \varphi_{1 j\left(t_{0}\right)}, \theta_{1 j\left(t_{0}\right)}$ represent the actual Relational Space (Persistence, index of complex, strategic valence) of each Duet at the initial time; $\rho_{1 j\left(t_{0}+\Delta t\right)}^{\circ}, \varphi^{\circ}{ }_{1 j\left(t_{0}+\Delta t\right)}^{\circ}, \theta_{1 j\left(t_{0}+\Delta t\right)}^{\circ}$ are their corresponding incipient derivatives.

In fact, in this case, the Interaction between the Initial System (in its original integrity) and the System after the effects of the earthquake, gives origin to a New Ordinal System, whose final configuration is represented in Table 6.

\section{Analysis of the Results of the Simulation of the Previous Inter-Action Processes}

On the basis of the results in Table 6, which refer to the Final Configuration of the System as a consequence of the earthquake, it is possible to recognize that such an Ordinal Exit represents a clear manifestation of the recovery of the Internal Stability on behalf of the System, as explicitly foreseen by Equation (2) of the MOP.

In fact, even at a first analysis, although extremely synthetic, it is possible to immediately recognize the reduction and mitigation of the effects of the earthquake, with reference to the values of the three Indicators, both with respect to their maximum values and their minimum values respectively.

For the sake of brevity, but also for clarity, the corresponding "mitigations" of the effects are reproduced here below in the form of percentage changes:

$\begin{array}{ccccc}\text { High Values of Persistence: } & \text { the value of } & -4.82 \% & \text { becomes } & -2.30 \% \\ \text { of Complex: } & \text { the value of } & -11.54 \% & \text { becomes } & -5.82 \% \\ \text { of Valence: } & \text { the value of } & -5.49 \% & \text { becomes } & -2.68 \% \\ \text { Low Values of Persistence: } & \text { the value of } & -9.92 \% & \text { becomes } & -4.95 \% \\ \text { of Complex: } & \text { the value of } & -6.53 \% & \text { becomes } & -3.19 \% \\ \text { of Valence: } & \text { the value of } & -5.50 \% & \text { becomes } & -2.68 \%\end{array}$

It is also evident that an organic picture of the results can be obtained through a more accurate and articulated comparison between the values in Table 5 and Table 6, respectively.

Nonetheless, the previous results unable us to surely affirm that the System manifests a higher Resistance with respect to the corresponding condition characterized by a total absence of an Ordinal Requalification.

At the same time, it is also possible to recognize a correlative increase of its Resilience.

In fact, in the presence of a prior Ordinal Requalification, the System, after having mitigated the effects of the earthquake, still keeps an Ordinality level sufficiently high to progressively reacquire its specific Role, both with respect to its Ordinal Relationships with its Habitat and, even more, with respect to the Ordinal Relations within itself. 
Table 6. Final reconfiguration of the urban system and correlative "mitigation" of the effects of the earthquake.

\begin{tabular}{|c|c|c|c|c|c|c|c|c|c|}
\hline & $\mathrm{Bl}$ & $\mathrm{Cl}$ & $\mathrm{El}$ & $\rho_{1 j\left(t_{0}\right)}$ & $\varphi_{1 j\left(t_{0}\right)}$ & $\theta_{1 j\left(t_{0}\right)}$ & $\rho_{1 j\left(t_{0}+\Delta t\right)}^{\circ}$ & $\varphi_{1 j\left(t_{0}+\Delta t\right)}^{\circ}$ & $\theta_{1 j\left(t_{0}+\Delta t\right)}^{\circ}$ \\
\hline 1 & 1 & 0 & 0.3570 & 0.7458 & 1.0573 & 0.3033 & 0 & 0 & 0 \\
\hline 2 & 1 & 0 & 0.6877 & 0.7831 & 1.9396 & 0.5842 & 0 & 0 & 0 \\
\hline 3 & 1 & 0 & 1.0183 & 0.8223 & 2.7353 & 0.8651 & 0 & 0 & 0 \\
\hline 4 & 1 & 0 & 1.3490 & 0.8635 & 3.4507 & 1.1461 & 0 & 0 & 0 \\
\hline 5 & 1 & 0 & 1.6797 & 0.9068 & 4.0917 & 1.4270 & 0 & 0 & 0 \\
\hline 6 & 1 & 0 & 2.0104 & 0.9522 & 4.6637 & 1.7080 & 0 & 0 & 0 \\
\hline 7 & 1 & 0 & 2.3411 & 0.9998 & 5.1718 & 1.9889 & 0 & 0 & 0 \\
\hline 8 & 1 & 0 & 2.6718 & 1.0499 & 5.6209 & 2.2699 & 0 & 0 & 0 \\
\hline 9 & 1 & 0 & 3.0025 & 1.1025 & 6.0154 & 2.5508 & 0 & 0 & 0 \\
\hline 10 & 1 & 0 & 3.3332 & 1.1577 & 6.3595 & 2.8318 & 0 & 0 & 0 \\
\hline 11 & 1 & 0 & 3.6639 & 1.2157 & 6.6571 & 3.1127 & 0 & 0 & 0 \\
\hline 12 & 1 & 0 & 3.9946 & 1.2765 & 6.9118 & 3.3936 & 0 & 0 & 0 \\
\hline 13 & 1 & 0 & 4.3253 & 1.3405 & 7.1271 & 3.6746 & 0 & 0 & 0 \\
\hline 14 & 1 & 0 & 4.6560 & 1.4076 & 7.3062 & 3.9555 & 0 & 0 & 0 \\
\hline 15 & 1 & 0 & 4.9867 & 1.4781 & 7.4520 & 4.2365 & 0 & 0 & 0 \\
\hline 16 & 1 & 0 & 5.3174 & 1.5521 & 7.5672 & 4.5174 & 0 & 0 & 0 \\
\hline 17 & 1 & 0 & 5.6481 & 1.6298 & 7.6546 & 4.7984 & 0 & 0 & 0 \\
\hline 18 & 1 & 0 & 5.9788 & 1.7114 & 7.7163 & 5.0793 & 0 & 0 & 0 \\
\hline 19 & 1 & 0 & 6.3095 & 1.7971 & 7.7548 & 5.3603 & 0 & 0 & 0 \\
\hline 20 & 1 & 0 & 6.6401 & 1.8871 & 7.7721 & 5.6412 & 0 & 0 & 0 \\
\hline 21 & 1 & 0 & 6.9708 & 1.9816 & 7.7701 & 5.9222 & 0 & 0 & 0 \\
\hline 22 & 1 & 0 & 7.3015 & 2.0808 & 7.7506 & 6.2031 & 0 & 0 & 0 \\
\hline 23 & 1 & 0 & 7.6322 & 2.1850 & 7.7153 & 6.4840 & 0 & 0 & 0 \\
\hline 24 & 1 & 0 & 7.9629 & 2.2944 & 7.6657 & 6.7650 & 0 & 0 & 0 \\
\hline 25 & 1 & 0 & 8.2936 & 2.4093 & 7.6034 & 7.0459 & 0 & 0 & 0 \\
\hline 26 & 1 & 0 & 8.6243 & 2.5300 & 7.5295 & 7.3269 & 0 & 0 & 0 \\
\hline 27 & 1 & 0 & 8.9550 & 2.6567 & 7.4454 & 7.6078 & 0 & 0 & 0 \\
\hline 28 & 1 & 0 & 9.2857 & 2.7897 & 7.3522 & 7.8888 & 0 & 0 & 0 \\
\hline 29 & 1 & 0 & 9.6164 & 2.9294 & 7.2510 & 8.1697 & 0 & 0 & 0 \\
\hline 30 & 1 & 0 & 9.9471 & 3.0761 & 7.1426 & 8.4507 & 0 & 0 & 0 \\
\hline 31 & 1 & 0 & 10.2778 & 3.2301 & 7.0282 & 8.7316 & 0 & 0 & 0 \\
\hline 32 & 1 & 0 & 10.6085 & 3.3919 & 6.9084 & 9.0126 & 0 & 0 & 0 \\
\hline 33 & 1 & 0 & 10.9392 & 3.5617 & 6.7840 & 9.2935 & 0 & 0 & 0 \\
\hline 34 & 1 & 0 & 11.2699 & 3.7400 & 6.6558 & 9.5744 & 0 & 0 & 0 \\
\hline 35 & 1 & 0 & 11.6006 & 3.9273 & 6.5244 & 9.8554 & 0 & 0 & 0 \\
\hline
\end{tabular}




\section{Continued}

\begin{tabular}{llllllllll}
\hline 36 & 1 & 0 & 11.9312 & 4.1240 & 6.3904 & 10.1363 & 0 & 0 & 0 \\
37 & 1 & 0 & 12.2619 & 4.3305 & 6.2544 & 10.4173 & 0 & 0 & 0 \\
38 & 1 & 0 & 12.5926 & 4.5473 & 6.1168 & 10.6982 & 0 & 0 & 0 \\
\hline
\end{tabular}

Captions: $\mathrm{Bl}, \mathrm{Cl}, \mathrm{Dl}$ are internal control parameters of the simulator EQS; $\rho_{1 j\left(t_{0}\right)}, \varphi_{1 j\left(t_{0}\right)}, \theta_{1 j\left(t_{0}\right)}$ represent the actual Relational Space (Persistence, index of complex, strategic valence) of each Duet at the initial time; $\rho_{1 j\left(t_{0}+\Delta t\right)}^{\circ}, \varphi_{1 j\left(t_{0}+\Delta t\right)}^{\circ}, \theta_{1 j\left(t_{0} \Delta t\right)}^{\circ}$ are their corresponding incipient derivatives.

\section{Conclusions}

The conclusions of the present analysis can be thus synthesized as follows:

- In view of possible Calamities (Earthquakes, Hurricanes, Floods, etc.);

- Any Urban-Territorial Reality should provide, in advance, to its Ordinal Requalification, appropriately commensurate to the "foreseeable" Calamities pertaining its specific case;

- This is because, from such an Ordinal Requalification, it will result a "Rebound" of its "Resistance" and at the same time, of its correlative level of "Resilience".

In this sense, the paper represents the basis for a consequential proposal to mayors and administrators of Urban Realities situated in areas characterized by a high seismic risk.

\section{Conflicts of Interest}

The authors declare no conflicts of interest regarding the publication of this paper.

\section{References}

[1] Samuele Biondi, S., Valter Fabietti, V. and Vanzi, I. (2011) Modelli di valutazione per la vulnerabilità sismica urbana. Magazine Urbanistica n. 147, University "G. d'Annunzio", Chieti, Italy.

[2] Giannantoni, C. (2010) The Maximum Ordinality Principle. A Harmonious Dissonance. Proceedings of the 6 th Emergy Conference, Gainesville, USA, 14-16 January 2010, 55-72.

[3] Giannantoni, C. (2012) The Relevance of Emerging Solutions for Thinking, Decision Making and Acting. The Case of Smart Grids. Ecological Modelling, 271, 62-71. https://doi.org/10.1016/j.ecolmodel.2013.04.001

[4] Giannantoni, C. (2014) Toward One Sole Reference Principle Generating "Emerging Solutions" of Progressively Ascending Ordinality. Proceedings of the $8^{\text {th }}$ Biennial Emergy Research Conference, Univ. of Florida, Gainesville (USA), 16-18 January 2014, 33-50.

[5] Giannantoni, C. (2016) The "Emerging Quality" of Self-Organizing Systems, When Modeled According to the Maximum Ordinality Principle, Offers a Radically New Perspective to Modern Science. Proceedings of the 9th Biennial Emergy Research Conference, Gainesville (USA), 6-7 January 2016, 281-298.

[6] Giannantoni, C. (2001) The Problem of the Initial Conditions and Their Physical Meaning in Linear Differential Equations of Fractional Order. Applied Mathematics 
and Computation, 141, 87-102. https://doi.org/10.1016/S0096-3003(02)00323-5

[7] Giannantoni, C. (2004) Mathematics for Generative Processes: Living and Non-Living Systems. Journal of Computational and Applied Mathematics, 189, 324-340. https://doi.org/10.1016/j.cam.2005.03.032

[8] Giannantoni, C. (2018) Self-Organizing Systems, When Modeled According to the Maximum Ordinality Principle, Always Present Explicit Formal Solutions, in Their Proper Time and Proper Space. Proceedings of the 10th Biennial Emergy Conference, University of Florida, Gainesville (USA), 25-27 January 2018, 137-156. 


\section{Appendix: General Explicit Solution to the Two Fundamental Equations of the MOP, Understood as a Whole}

The first Fundamental Equation (1) always presents an explicit solution [8], which can always be written in the general form

$$
\{\tilde{r}\}=e^{\{\tilde{\alpha}(t)\}}=e^{\left\{\left(\begin{array}{c}
\tilde{\alpha}_{11}(t) \\
\tilde{\alpha}_{21}(t) \\
\vdots \\
\tilde{\alpha}_{N 1}(t)
\end{array}\right),\left(\begin{array}{c}
\tilde{\alpha}_{12}(t) \\
\tilde{\alpha}_{22}(t) \\
\vdots \\
\tilde{\alpha}_{N 2}(t)
\end{array}\right)\left(\begin{array}{c}
\ldots \\
\cdots \\
\cdots \\
\cdots
\end{array}\right)\left(\begin{array}{c}
\tilde{\alpha}_{1 N}(t) \\
\tilde{\alpha}_{2 N}(t) \\
\vdots \\
\tilde{\alpha}_{N N}(t)
\end{array}\right)\right\}}
$$

where the Relational Space $\{\tilde{r}\}=\mathrm{e}^{\{\tilde{\alpha}(t)\}}$ depends on the Nature of the System analyzed, while the corresponding structure of each term of the Ordinal Matrix depends on the Specific Generativity $(\underline{\tilde{d} / \tilde{d} t})_{s}$.

For example, if the Relational Space of the System is represented by $\{\tilde{\rho}, \tilde{\varphi}, \tilde{\vartheta}\}$, that is three topological coordinates always considered as the exit of a Generative Process, we can assume

$$
\{\tilde{r}\}_{S}=e^{\tilde{\alpha}(t)}=e^{\{\tilde{\sigma} \circledast \tilde{i} \oplus \tilde{\varphi} \circledast \tilde{j} \oplus \tilde{\vartheta} \circledast \tilde{k}\}}
$$

This is because, on the basis of a generalized form of De Moivre representation, it is always possible to write

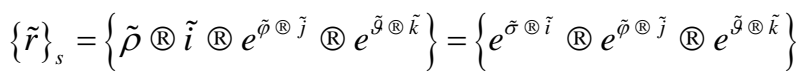

$$
\begin{aligned}
& =e^{\{\tilde{\sigma} \circledast \tilde{i} \oplus \tilde{\varphi} \circledast \tilde{j} \oplus \tilde{\vartheta} \circledast \tilde{k}\}}=e^{\tilde{\alpha}(t)}
\end{aligned}
$$

where the traditional versors $\vec{i}, \vec{j}, \vec{k}$ are now replaced by three unit spinors $\tilde{i}, \tilde{j}, \tilde{k}$, which are defined in such a way as to satisfy the following Relational Product Rules:

$$
\begin{aligned}
& \tilde{i}{ }^{\circledR} \tilde{i}=\oplus 1, \tilde{i}{ }^{\circledR} \tilde{j}=\tilde{j}, \tilde{i}{ }^{\circledR} \tilde{k}=\tilde{k} \\
& \tilde{j}{ }^{\circledR}=\tilde{j}, \tilde{j}{ }^{\circledR} \tilde{j}=\Theta 1, \tilde{j}{ }^{\circledR} \tilde{k}=\tilde{k} \\
& \tilde{k}{ }^{\circledR}=\tilde{k}, \tilde{k}{ }^{\circledR} \tilde{j}=\tilde{k}, \quad \tilde{k}{ }^{\circledR} \tilde{k}=\Theta 1
\end{aligned}
$$

where the symbols $\oplus$ and $\left({ }^{B}\right.$ express more intimate relationships between the same: both in terms of sum $(\oplus)$ and in terms of (relational) product $(\circledR)$ with respect to the case of traditional versors $\vec{i}, \vec{j}, \vec{k}$.

So that representation (A3) is similar (albeit not strictly equivalent) to a system of three complex numbers, characterized by one real unit $(\tilde{i})$ and two imaginary units $(\tilde{j}$ and $\tilde{k})$.

Equation (A1) thus describes the Generative Evolution of the System as the exit of an Ordinal Cooperation of $\mathrm{NCo-Productions} \mathrm{(vertical} \mathrm{brackets)} \mathrm{and} \mathrm{their}$ associated $N$ Inter-Actions (horizontal brackets). At the same time, when the Process has reached its Maximum Ordinality, each term $\tilde{\alpha}_{i j}(t)$ of the Ordinal Matrix, as a consequence of such a Maximization Process, will transform into a binary-duet Relationship, represented as $\left\{\tilde{\alpha}_{i j}(t)\right\}^{\{\tilde{2} / \tilde{2}\}}$.

At the same time, the adoption of the brackets " \{\} " in Equation (A1) is explicitly finalized to remind us that the Ordinal Matrix represents the Ordinal Structure of the System understood as a Whole. 
In fact, all the elements of the Ordinal Matrix (in Equation (A1)) satisfy the following "Ordinal Relationships"

$$
\begin{aligned}
& \left.\left\{\tilde{\alpha}_{i, j+1}(t)\right\}^{\{\tilde{2} / 2}\right\} \oplus\left\{\tilde{\lambda}_{i, j+1}(t)\right\}^{\{\tilde{z} / \tilde{2}\}} \\
& \stackrel{*}{=}(\sqrt[N-1]{\{\tilde{1}\}})_{j}{ }^{\circledR}\left\{\tilde{\alpha}_{12}(t)\right\}^{\{\tilde{z} / \tilde{2}\}} \oplus\left\{\tilde{\lambda}_{12}(t)\right\}^{\{\tilde{z} / \tilde{z}\}} \text { for } j=1,2,3, \cdots, N-1
\end{aligned}
$$

where the additional terms $\left\{\tilde{\lambda}_{i, j}(t)\right\}^{\{\tilde{2} / \tilde{2}\}}$ explicitly account for the associated habitat conditions.

Equations (A5) can also be termed as "Harmony Relationships" precisely because they show that all the elements $\left.\left.\left\{\tilde{\alpha}_{i, j+1}(t)\right\}^{\{\tilde{z} / 2}\right\} \oplus\left\{\tilde{\lambda}_{i, j+1}(t)\right\}^{\{\tilde{2} / 2}\right\}$ of the Ordinal Matrix can be obtained by means of one sole arbitrary couple (Duet) $\left\{\tilde{\alpha}_{12}(t)\right\}^{\{\tilde{2} / \tilde{2}\}} \oplus\left\{\tilde{\lambda}_{12}(t)\right\}^{\{\hat{2} / 2\}}$, assumed as reference, and the N-1 Ordinal Roots $(\sqrt[N-1]{\{\tilde{1}\}})_{j}$ of Unity $\{\tilde{1}\}$.

Consequently, if each element of the Ordinal Matrix (in Equation (A1)) is expressed in terms of the reference couple $\left.\left\{\tilde{\alpha}_{12}(t)\right\}^{\{\tilde{2} / 2}\right\} \oplus\left\{\tilde{\lambda}_{12}(t)\right\}^{\{\hat{2} / 2)}$, the solution to the first Fundamental Equation (1) assumes the following form (Equation (A6))

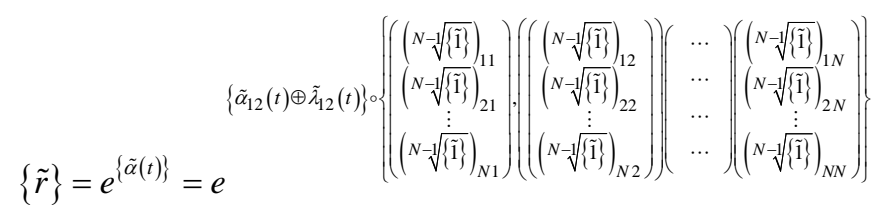

where, for the sake of simplicity, the term $\left\{\tilde{\alpha}_{12}(t) \oplus \tilde{\lambda}_{12}(t)\right\}$ stands for $\left.\left\{\tilde{\alpha}_{12}(t)\right\}^{\{\tilde{2} / 2}\right\} \oplus\left\{\tilde{\lambda}_{12}(t)\right\}^{\{\tilde{2} / \tilde{2}\}}$.

The same Ordinal Matrix, in addition, may always be represented in a more synthetic form by means of one sole symbol by adopting the following synthetic notation

$$
\left\{(\sqrt[N-1]{\{\tilde{1}\}})_{i j}\right\}^{\uparrow\{\tilde{N} / \tilde{N}\}}
$$

where the arrow " $\uparrow$ ” explicitly reminds us that the Ordinality $\{\tilde{N} / \tilde{N}\}$ has always to be considered as being a particular form of Over-Ordinality.

In this way the explicit solution to the first Fundamental Equation (1) can synthetically be expressed as follows

$$
\{\tilde{r}\}=e^{\{\tilde{\alpha}(t)\}}=e^{\left\{\tilde{\alpha}_{12}(t) \oplus \tilde{1}_{12}(t)\right\} \cdot(N-\sqrt{(\tilde{i})})^{\uparrow(\tilde{N} / \tilde{N}\}}}
$$

Consequently, if such a solution is introduced into the Global Feed-Back Process represented by the second Fundamental Equation (2), the latter transforms into a typical Riccati's Equation of Ordinal Nature, whose explicit solution is given by

$$
\{\tilde{r}\}=e^{\{\tilde{\alpha}(t)\}}=e^{\{\tilde{B}(t)\} ?(N-\sqrt[N]{\{(\tilde{i}\}})^{\uparrow\{\tilde{N} / \tilde{N}\}}}
$$


where

$$
\tilde{B}(t)=\left\{\left(\begin{array}{l}
\oplus \tilde{A}(t) \\
\Theta \tilde{A}(t)
\end{array}\right),\left(\begin{array}{c}
\Theta \tilde{A}(t) \\
\oplus \tilde{A}(t)
\end{array}\right)\right\}
$$

and

$$
\tilde{A}(t)=\left\{\left\{\tilde{\alpha}_{12}(0)\right\}^{\{\tilde{2} / \tilde{2}\}} \oplus\left\{\tilde{\lambda}_{12}(0)\right\}^{\{\tilde{z} / \tilde{2}\}}\right\} \circ\left\{(\sqrt[N-1]{\{\tilde{1}\}})^{\uparrow\{\tilde{N} / \tilde{N}\}}\right\}^{(\tilde{2} / \tilde{2})} \oplus \ln \left(\tilde{c}_{1} \oplus\left\{\tilde{c}_{2}, t\right\}\right)
$$

in which the term $\ln \left(\tilde{c}_{1} \oplus\left\{\tilde{c}_{2}, t\right\}\right)$ accounts for the origin and habitat conditions of the Feed-Back Equation and, at the same time, also represents an Over-Ordinality contribution specifically due to the same Feed-Back Process.

Equation (A9) then represents the Explicit "Emerging Solution" to the Maximum Ordinality Principle, formulated in two "Incipient" Differential Equations ((1) and (2)), considered as being a Whole. 